Editorial

\title{
Pattern Recognition in Remote Sensing
}

Development of new pattern recognition techniques for the analysis of data collected from satellites and airborne sensors used for Earth observation has been a popular research topic for several decades. Consequently, the pattern recognition and remote sensing research fields have always overlapped. However, the large volumes of remote sensing data acquired from last generation sensors require new advanced algorithms and techniques for automatic analysis. This data volume, together with new applications ranging from monitoring of human settlements to management of natural resources, from agricultural studies to response for natural and human-induced disasters, from the assessment of the impact of climate change to conserving biodiversity, require new interdisciplinary work involving the application of novel pattern recognition techniques to unsolved problems in remote sensing image analysis that cannot be handled by using traditional remote sensing methods.

One of the most important challenges is the increasing resolution of the data, leading to an expansion in the data volume and an increase in the complexity of the analysis algorithms. Higher resolution often means that additional patterns are visible in large scenes and, therefore, more elaborate yet faster techniques need to be developed to detect and recognize them. Furthermore, a characteristic peculiar to remote sensing is that "high" resolution may mean not only "high spatial", but also "high spectral" resolution, leading to a wealth of problems related to high dimensionality of feature spaces, and "high temporal" resolution, requiring new methods for time series analysis. Researchers also need to take into account the nature of different sensors used for collecting data with different modalities such as multi-spectral and hyper-spectral data, synthetic aperture radar (SAR) data, and light detection and ranging (LIDAR) data for developing proper techniques capable to model the peculiar statistical properties of each type of data in the implemented methodologies. Finally, performance evaluation of the developed supervised, semi-supervised, and unsupervised algorithms is also an interesting problem given the limited availability of detailed ground truth data sets.

This special issue is associated with the 5th IAPR Workshop on Pattern Recognition in Remote Sensing (PRRS 2008) that was held in Tampa, FL, USA on December 7, 2008 in conjunction with the IAPR International Conference on Pattern Recognition (ICPR 2008) with co-sponsorship by IAPR and IEEE Geoscience and Remote Sensing Society. The PRRS Workshop which is implemented by the IAPR Technical Committee 7 on Remote Sensing offers an opportunity for researchers to gain a better understanding of the many diverse research topics in remote sensing that require contributions from the pattern recognition community, and has established itself as an important event for scientists involved in the combined fields of pattern recognition and remote sensing.
This special issue follows the tradition of two earlier issues (Gamba and Clausi, 2006; Clausi et al., 2007) for promoting state-of-the-art pattern recognition research on the analysis of multi-spectral, multi-modal, multi-resolution, and multi-temporal remote sensing data sets. Papers were solicited from participants in the PRRS Workshop and from the pattern recognition, geoscience, and remote sensing communities at large through an open call for papers. The issue is composed of 15 papers, selected among the 23 received submissions according to the standard review process of Pattern Recognition Letters, covering many different aspects of remote sensing image analysis.

The first part of the special issue contains contributions related to object detection techniques designed for different types of remote sensing data. The paper by Michaelsen et al. describes an implementation of the grouping and decision layers of a Gestalt system for detecting closed polygons delineating building outlines in SAR images. The paper by Wang et al. proposes a new approach at the rectangle feature level to extract buildings from high-resolution polarimetric synthetic aperture radar (PolSAR) data, using both region-based and edge-based information. The paper by Bartels and Wei presents a two-step approach for object and ground point separation in airborne LIDAR data. The first step categorizes LIDAR tiles as hilly or moderate terrains, and the second step adapts the skewness balancing method for unsupervised classification to different terrain types. The paper by Yao et al. addresses the vehicle extraction problem for traffic monitoring in urban areas using airborne laser scanning (ALS) data. The proposed contextguided approach is based on a geometric model of vehicles where ground separation is used to exclude irrelevant objects and watershed segmentation is used to isolate the vehicles. Erus and Lomenie have developed a decision making system that recognizes and detects complex man-made objects like bridges or roundabouts from very high resolution satellite imagery. The hybrid approach is based on learning the spatial configuration of structural primitives constituting an object, according to their statistical distribution in a labeled training set. The paper by Valero et al. presents a new method for extracting roads in very high resolution remotely sensed images based on advanced directional morphological operators called path openings and path closings. The paper by Ozdemir et al. proposes a new procedure for quantitative evaluation of object detection algorithms. The procedure consists of a matching stage for finding correspondences between reference and output objects, an accuracy score that is sensitive to object shapes as well as boundary and fragmentation errors, and a ranking step for final ordering of the algorithms using multiple performance indicators.

The second part of the special issue addresses the important and challenging problem of change detection in multi-temporal 
remote sensing images. The paper by Champion et al. describes an automatic method for detecting changes in a 2D building database using recent satellite images. The method uses 3D primitives that are collected for each building and are matched with primitives derived from the existing database for acceptance or rejection of changes. The paper by Bovolo et al. presents a novel method for change detection based on change vector analysis and support vector data description technique. This method formulates the change detection problem in multi-temporal remote sensing images as a minimum enclosing ball problem with changed pixels as target objects. The authors provide experimental results that show a better identification of changed areas with respect to other techniques in the literature, particularly in problems with overlapping classes. Sirmacek and Unsalan have developed an automated method to measure land development in a given urban region imaged at different times. Their approach takes into consideration the local features extracted using Gabor filtering and local maxima detection. Experimental results on high resolution panchromatic imagery indicate an improvement in performance and low computational cost compared to a previous approach in the literature.

The third part of the special issue is focused on specific data analysis techniques associated to different remote sensing data and problems. The paper by Wong and Clausi presents an automated system named AISIR (Automated Inter-sensor/Inter-band Satellite Image Registration) that has been designed and implemented for registering satellite images acquired using different sensors and spectral bands. It introduces a robust mapping function estimation scheme based on the proposed modified GemanMcClure M-estimation scheme. The system has been tested using multi-spectral optical, LIDAR, and SAR images and is shown to achieve better registration accuracy than other well known registration schemes. Kerroum et al. present a new method for feature selection, based on the Gaussian mixture model, for estimating Shannon's mutual information between multiple features and the output class labels from limited training samples. They show that this method not only improves the classification performance, but also can be very useful to avoid the Hughes effect by eliminating irrelevant features for remotely sensed imagery. The paper by Bandeira et al. proposes a multi-layer strategy to extract topological features of large polygonal networks observed on remotely sensed images of Mars. The paper by Turlapaty et al. presents a method to fuse several precipitation datasets by using artificial neural networks and vector space transformation function. The paper by Lampert and O'Keefe presents an active contour framework with application to the detection of single and multiple tracks in spectrograms. To simplify the problem, the authors use a potential model that combines the tracking and detection process into one stage. This results in feature extraction at a Signal-to-Noise Ratio (SNR) as low as $0.5 \mathrm{~dB}$.

We are pleased to offer this special issue to the pattern recognition and remote sensing scientific communities, and hope that it accomplishes our goal of highlighting recent advances in a wide range of challenges within the combined fields of pattern recognition and remote sensing.

\section{References}

Proceedings of 2008 IAPR Workshop on Pattern Recognition in Remote Sensing. <http://www.ieeexplore.ieee.org/servlet/opac?punumber=4777931>.

Gamba, P., Clausi, D., 2006. Preface. Pattern Recognition Lett. 27 (4), 217.

Clausi, D.A., Aksoy, S., Tilton, J.C., 2007. Foreword to the Special Issue on Pattern Recognition in Remote Sensing. IEEE Trans. Geosci. Remote Sens. 45 (12), 38553856.

Selim Aksoy

Department of Computer Engineering, Bilkent University, Bilkent, 06800 Ankara,

Turkey

Tel.: +90 312 2903405; fax: +903122664047

E-mail address: saksoy@cs.bilkent.edu.tr

Nicolas H. Younan

Department of Electrical and Computer Engineering, Mississippi State University, Mississippi State, MS,

Tel.: +1 6623253721

E-mail address: younan@ece.msstate.edu

Lorenzo Bruzzone

Department of Information Engineering and Computer Science, University of Trento, I-38123 Trento,

Italy

Tel.: +390461882056

E-mail address: lorenzo.bruzzone@ing.unitn.it

Available online 24 April 2010 